\title{
ESTIMATION OF UNCERTAINTY OF TRYPSIN INHIBITOR ACTIVITY MEASUREMENT IN LEGUME CROPS
}

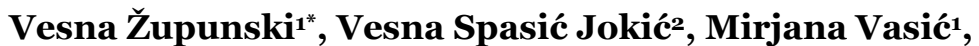 \\ Aleksandra Savić1, Zoran Mitrovićé, Ivan Župunski ${ }^{2}$ \\ ${ }^{1}$ Institute of Field and Vegetable Crops, Novi Sad, Serbia \\ ${ }^{2}$ Faculty of Technical Sciences, University of Novi Sad, Novi Sad, Serbia
}

\begin{abstract}
Irradiation of legume seeds has emerged as an attractive alternative compared to conventional chemical treatments in seed production. Irradiation is also used for the decontamination of food and feed in order to increase the shelf-life of fresh and dry food materials. The effects of irradiation on nutritive and anti-nutritive factors such as trypsin inhibitors are usually reported together with the measurements obtained by using the quantitative analytical methods. The objective of this study was to measure trypsin inhibitor activity (TIA) of common bean cultivar Oplenac using the microtiter plate method and to identify factors that contribute to the uncertainty of TIA measurement according to the current Guide to the Expression of uncertainty in measurement (GUM). Dominant sources of uncertainty of TIA measurement were: absorbance measurements of sample and positive control reaction mixtures and preparation of the final sample solution using a graduated cylinder (V4). Absorbance measurement of sample reaction mixtures took $37.8 \%$ of the overall measurement uncertainty. Preparation of the final sample solution using a graduated cylinder (V4) and absorbance measurement of positive control reaction mixture contributed to the overall uncertainty with $35.1 \%$ and $15.8 \%$, respectively. Acquired insight into factors that contribute to the uncertainty of TIA measurement gives directions for the improvement of TIA testing methods and TIA results management.
\end{abstract}

Key words: Measurement uncertainty, trypsin inhibitor activity, common bean

DOI: $10.21175 /$ RadProc.2016.38

\section{INTRODUCTION}

Irradiation of legume seeds has emerged as an attractive alternative when compared to conventional chemical treatments used to minimize losses that occur during seed storage and production. In addition, ionizing radiation has been used as another technique for decontamination of food and feed in order to increase the shelf-life of fresh and dry food materials [1], [2], [3]. As a consequence, the effects of an irradiation on nutritional characteristics of food and feed materials were investigated in many studies.

Legume crops are of great concern to food and feed industry as an important source of proteins, carbohydrates, fats, fibers, essential vitamins, and minerals. The presence of anti-nutritional factors, such as protease inhibitors, could decrease nutritional value of legume crops and limit their utilization in human and animal nutrition, but could also be beneficial to human health by preventing diseases such as cancer [4], [5], [6]. Depending on time and dose of consumption, protease inhibitor will have beneficial or anti nutritional effects [7]. As a consequence, exploration of protease inhibitors, especially of trypsin inhibitors is of great concern, and in some countries allowed trypsin inhibitor activity (TIA) of new legume cultivars is statutorily prescribed. Since it was reported that irradiation could have effects on nutritional characteristics of food and feed, a number of investigations were conducted in order to estimate the irradiation effects on anti-nutritional factors such as tryspin inhibitors.

Considerable increase in protein values, and decrease in TIA during germination of irradiated green grams was reported by [8]. Significant linear relationships have been reported in chick pea between the loss of TIA and increasing radiation dose (0.25$1.00 \mathrm{kGy}$ ) with little or no effect on protein content [9]. The loss of TIA was found to be $54.5 \%$ when soybeans were subjected to $10 \mathrm{kGy}$ [10]. According to Serbian national legislation [11] and Directive 1999/2/EC of the European Parliament concerning foods and food ingredients treated with ionising radiation [12], legume crops may be treated with ionising radiation with doses less than $1 \mathrm{kGy}$. The effects of irradiation on nutritive and anti-nutritive components of legumes are usually reported together with measurements obtained by using the quantitative analytical methods.

Methods for TIA measurement are based on the hydrolysis of Na-Benzoyl-,L-arginine 4-nitroanilide hydrochloride (L-BAPNA) by trypsin and includes spectrophotometric measurement of the reaction

\footnotetext{
*vesnazup.nsseme@gmail.com
} 
products. Measurement results of TIA are usually accompanied with standard deviation, however, analysis of uncertainty sources of TIA measurement were not previously analyzed. In order to demonstrate the quality of measurement results it is important to estimate measurement uncertainty. Results accompanied with statement of measurement uncertainty increase confidence in the validity of a measurement results and enable comparisons between results obtained by different techniques or compliance with regulatory levels. The aim of this study was to estimate and to analyze uncertainty of TIA measurements using the microtiter plate method according to the concept of measurement uncertainty described in the current Guide to the Expression of uncertainty in measurement (GUM) [13].

\section{MATERIALS AND METHODS}

\subsection{Preparation of sample solution}

Starting material for TIA measurement was seed of common bean (Phaseolus vulgars), variety Oplenac, originating from the collections of the Institute of Field and Vegetable Crops, Novi Sad, Serbia. Extraction of trypsin inhibitors was performed using method reported by [14]. Trypsin inhibitors were extracted from the grounded raw seeds (0.2 g) using $20 \mathrm{~mL}$ of distilled water $\left(V_{1}\right)$. Obtained sample suspension were completed by adding $20 \mathrm{~mL}\left(V_{2}\right)$ of assay buffer (50 mM Tris buffer, $\mathrm{pH} 8.2$, containing $10 \mathrm{mM} \mathrm{CaCl}_{2}$ ), and after shaking for 2 -3 minutes it was filtered through a Whatman No. 2 paper (Sigma Aldrich, USA). After filtration, $1 \mathrm{~mL}$ of filtrate (initial sample solution, $V_{3}$ ) was additionally diluted with $6.5 \mathrm{~mL}$ of distilled water $\left(V_{4}\right)$ in order to obtain final sample solution which would give $30 \%-70 \%$ of trypsin inhibition.

\subsection{TIA test}

TIA testing was carried out using microtiter plate method with assay conditions described by [14]. Reaction mixtures of sample, positive and negative controls were set up in each microtiter plate row. Reaction mixtures were set up by mixing: $45-\mu$ l of the final sample solution or distilled water (for control reaction mixtures), $22.5 \mu \mathrm{l}$ of trypsin solution and $90 \mu \mathrm{l}$ of L-BAPNA solution. Pre-incubation of final sample solution or distilled water with trypsin solution was performed at $37^{\circ} \mathrm{C}$ for 10 minutes, and after adding 90 $\mu \mathrm{l}$ of solution of L-BAPNA incubation was carried out for 30 minutes at $37{ }^{\circ} \mathrm{C}$. Positive control reaction mixture gave non-inhibited reaction of enzyme (trypsin) and substrate (L-BAPNA), while negative control reaction mixture was used as reagent blank, since $45 \mu \mathrm{l}$ of $30 \%$ acetic acid was added immediately after trypsin solution in order to stop reaction. The absorbances of reaction mixtures were measured by using Multiskan Ascent microtiter plate photometer (Thermo Fisher Scientific, California, USA).

TIA was calculated according to Equation 1 provided by [14]. The trypsin inhibitor activity was expresed in number of trypsin units inhibited (TIU) per miligram of seed sample, taking into account the fact that one trypsin unit is defined as an increase of 0.01 absorbance units at $405 \mathrm{~nm}$.

$$
T I A=\frac{\left(A_{p c}-A_{s}\right) \times 100}{m_{s}}
$$

where:

$m_{\mathrm{s}}$ - is mass (mg) of original material (seed) contained in $1 \mathrm{~mL}$ of final sample solution

$A_{p c}$ - absorbance measurement of the positive control reaction mixture

$A_{s}$ - absorbance measurement of the sample reaction mixture

\subsection{Uncertainty of TIA measurement}

A large number of experiments were conducted and uncertainty analysis was obtained using a procedure reported by [15].

The main sources of uncertainty of a TIA measurement are identified from measurement function (Equation 1) and they include: absorbance measurement of the sample and positive control reaction mixture and concentration of the original material in the final sample solution. As a result combined standard uncertainty of TIA was calculated as follows:

$u(T I A)=\sqrt{\left(\frac{\partial T I A}{\partial A_{p c}} u\left(A_{p c}\right)\right)^{2}+\left(\frac{\partial T I A}{\partial A_{s}} u\left(A_{s}\right)\right)^{2}+\left(\frac{\partial T I A}{\partial m_{s}} u\left(m_{s}\right)\right)^{2}}$

Factors that influence the uncertainty of the absorbance measurement of positive control reaction mixture ( $A \mathrm{pc}$ ) includes: correction due to the dispersion of absorbance measurement results of the positive control reaction mixture and the related standard uncertainty; correction due to the calibration of the photometer and the related standard uncertainty; correction due to the finite indication resolution of absorbance measurement of the positive control reaction mixture and related standard uncertainty; volume of trypsin solution; volume of LBAPNA solution; correction due to the variation of individually delivered volumes of trypsin solution using an automatic pipette and related standard uncertainty; correction due to the variation of individually delivered volumes of L-BAPNA solution using an automatic pipette and related standard uncertainty.

Similar to the previous estimation of the combined standard uncertainty of $A \mathrm{pc}$, the combined standard uncertainty of absorbance measurement of the sample reaction mixture $A$ s includes: correction due to the dispersion of absorbance measurement results of the sample reaction mixture and related standard uncertainty; correction due to the calibration of the photometer and the related standard uncertainty; correction due to the finite indication resolution of absorbance measurement of the sample reaction mixture and related standard uncertainty; correction due to the variation of individually delivered volumes of final sample solution using an automatic pipette and related standard uncertainty; correction due to the calibration of the automatic pipette used for the volume delivery of the final sample solution and correction due to the volume delivery variation of the final sample solution caused by temperature variation. 
Uncertainty sources related to the quantity $m_{s}$ are: correction due to the calibration of the analytical balance used for weighing of ground seed and related standard uncertainty; sensitivity coefficients, defined as the partial derivatives of function $m_{\mathrm{s}}$ of input quantities $x_{\mathrm{i}}$ ( $\mathrm{x}_{\mathrm{i}}$ are $m, V_{1}, V_{2}, V_{3}$ and $V_{4}$ ); combined standard uncertainty of delivered volumes; correction due to the calibration of the graduated cylinder used for delivery of the volume $V_{i}$ and associated standard uncertainty; correction due to the volume delivery $V_{i}$ variation caused by temperature variation and associated standard uncertainty; correction due to the variation of individually delivered volume $V_{i}$ and associated standard uncertainty.

\section{RESULTS AND DISCUSSION}

Measured TIA of common bean variety Oplenac was $56.2 \mathrm{TIU} / \mathrm{mg}$ and expanded measurement uncertainty (with coverage factor $k=2$ ) was aproxitemately $5.2 \mathrm{TIU} / \mathrm{mg}$ (9\%).

Dominant sources of uncertainty of TIA measurements were: absorbance measurements of the sample and the positive control reaction mixture, and preparation of the final sample solution using graduated cylinder $\left(V_{4}\right)$ (Fig. 1, Fig. 2).
Absorbance measurement of the sample reaction mixture $(A \mathrm{~s})$ took $37.8 \%$ of the overall uncertainty of TIA measurement with repeatability of absorbance measurement $(A \mathrm{~s}, \mathrm{~m})$ contributing dominantly to the uncertainty with $37 \%$. Volume delivery of the final sample solution $(V \mathrm{~s})$, trypsin solution $(V \mathrm{~s}, \mathrm{~T})$ and $\mathrm{L}-$ BAPNA solution $(V \mathrm{~s}, \mathrm{~B})$ using automated pipette, when the sample reaction mixtures were prepared, had smaller contribution to uncertainty with $4.6 \%$, $0.3 \%$ and $1.6 \%$, respectively. Absorbance measurement and preparation of sample reaction mixtures took the largest percent (44\%) of overall uncertainty of TIA value.

Absorbance measurement of positive control reaction mixture $(A \mathrm{pc})$ took $15.8 \%$ of $T I A$ measurement uncertainty with repeatability of absorbance measurement $(A \mathrm{pc}, \mathrm{m})$ contributing to uncertainty with $15.06 \%$. Volume delivery of the trypsin solution $(V \mathrm{pc}, \mathrm{T})$ and L-BAPNA solution $(V \mathrm{pc}, \mathrm{B})$ using automated pipette had contribution to uncertainty of TIA measurement with only $0.3 \%$ and $1.6 \%$, respectively. Absorbance measurement and preparation of positive control reaction mixtures had the smallest contribution to uncertainty of TIA measurement (18\%)

\begin{tabular}{|c|c|c|c|c|}
\hline $\begin{array}{c}\text { Sample mass } \\
m\end{array}$ & $\begin{array}{c}V 1 \\
\text { Using graduated } \\
\text { cylinder }\end{array}$ & $\begin{array}{c}\text { V2 } \\
\text { Using graduated } \\
\text { cylinder }\end{array}$ & $\begin{array}{c}\text { V3 } \\
\begin{array}{l}\text { Using automatic } \\
\text { pipette }\end{array}\end{array}$ & $\begin{array}{l}\text { V4 } \\
\text { Using graduated } \\
\text { cylinder }\end{array}$ \\
\hline \multirow[t]{3}{*}{ Scale accuraccy } & Repeatability & Repeatability & Repeatability & Repeatability \\
\hline & Temperature & Temperature & Temperature & Temperature \\
\hline & Calibration & Calibration & Calibration & Calibration \\
\hline \multicolumn{5}{|c|}{ Final sample solution preparation } \\
\hline \multicolumn{2}{|c|}{$\begin{array}{c}\text { Photometer } \\
\text { As }\end{array}$} & $\begin{array}{c}\text { Trypsin volume } \\
\text { Vs,T }\end{array}$ & $\begin{array}{c}\text { L-BAPNA volume } \\
\text { Vs,B }\end{array}$ & $\begin{array}{c}\text { Sample volume } \\
\text { Vs }\end{array}$ \\
\hline \multicolumn{2}{|c|}{ Repeatability } & Repeatability & Repeatability & Repeatability \\
\hline \multicolumn{2}{|c|}{ Resolution } & Temperature & Temperature & Temperature \\
\hline \multicolumn{2}{|c|}{ Calibration } & Calibration & Calibration & Calibration \\
\hline \multicolumn{2}{|c|}{$\begin{array}{l}\text { Absorbance } \\
\text { measurement }\end{array}$} & \multicolumn{3}{|c|}{$\begin{array}{l}\text { Sample reaction mixture preparation (filling of microtiter } \\
\text { plate using automatic pipette) }\end{array}$} \\
\hline \multicolumn{2}{|c|}{$\begin{array}{l}\text { Photometer } \\
\text { Apc }\end{array}$} & $\begin{array}{l}\text { Trypsin volume } \\
\qquad \mathrm{Vpc}, \mathrm{T}\end{array}$ & $\begin{array}{l}\text { L-BAPNA volume } \\
\qquad \text { Vpc, B }\end{array}$ & \\
\hline \multicolumn{2}{|c|}{ Repeatability } & Repeatability & Repeatability & \\
\hline \multicolumn{2}{|c|}{ Resolution } & Temperature & Temperature & \\
\hline \multicolumn{2}{|c|}{ Calibration } & Calibration & Calibration & \\
\hline \multicolumn{2}{|c|}{$\begin{array}{l}\text { Absorbance } \\
\text { measurement }\end{array}$} & \multicolumn{3}{|c|}{$\begin{array}{l}\text { Positive control reaction mixture preparation (filling of } \\
\text { microtiter plate using automatic pipette) }\end{array}$} \\
\hline
\end{tabular}

Figure 1. Sources of uncertainty of TIA measurement 


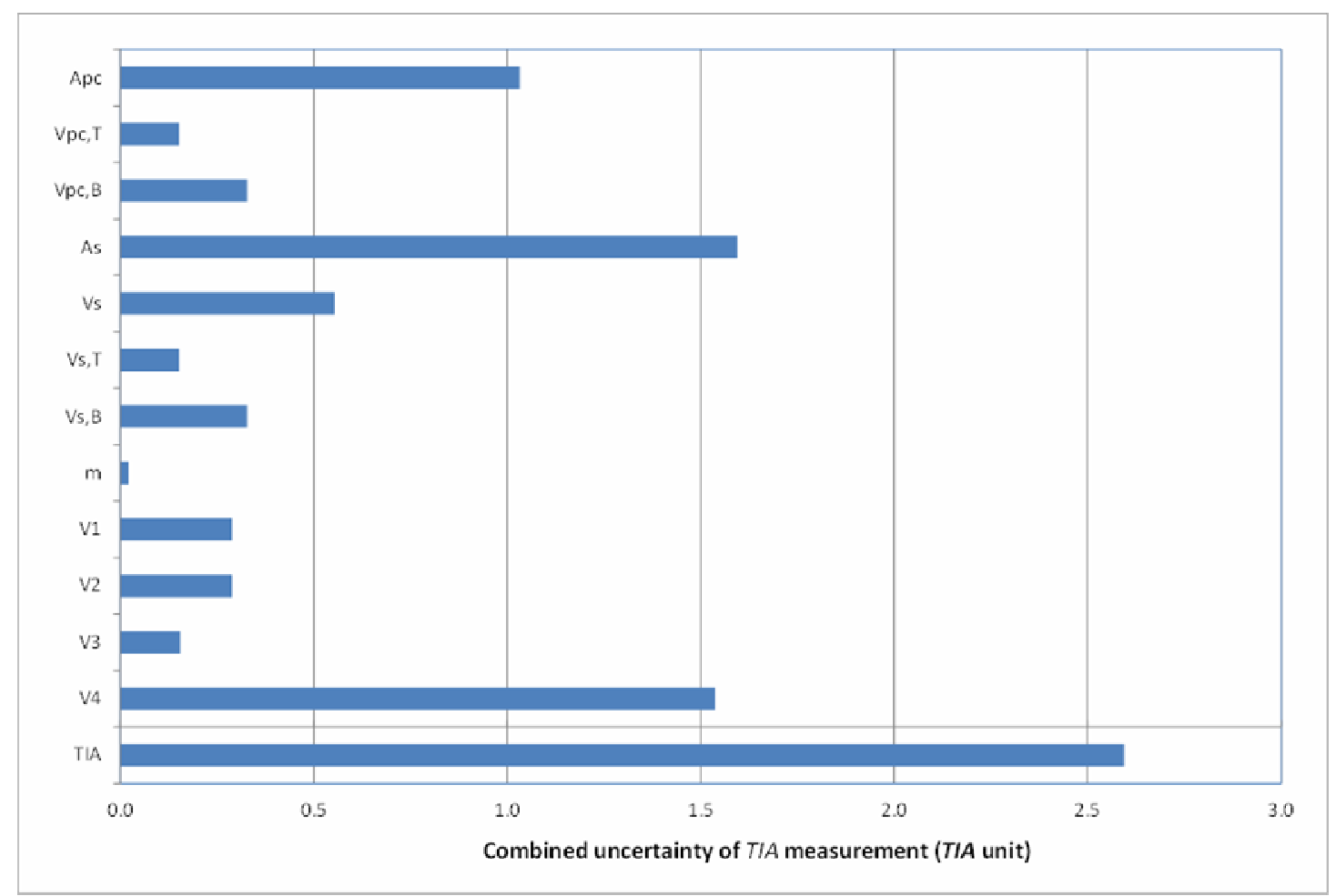

Figure 2. Contribution of uncertainty sources to combined uncertainty of TIA measurement

Preparation of the final sample solution considered using graduated cylinders ( $V_{1}, V_{2}$ and $\left.V_{4}\right)$ and automatic pipette $\left(V_{3}\right)$. Preparation of the final sample solution took $38 \%$ of overall uncertainty of TIA measurement. The dominant influence $(35.1 \%)$ had preparation of the final sample solution using graduated cylinder $\left(V_{4}\right)$ with correction due to calibration contributing to uncertainty with $34.8 \%$. Smaller contribution showed volume delivery of $V_{1}, V_{2}$ and $V_{3}$ with $1.2 \%, 1.2 \%$ and $0.4 \%$, respectively. Contribution of the preparation of the final sample solution to uncertainty of TIA measurement was almost equal to contribution of repeatability of absorbance measurement of sample reaction mixture (37\%) indicating that the preparation of the final sample solution had considerable impact on uncertainty and that it should be performed by using more accurate volumetric instruments than graduated cylinders are, or graduated cylinders should have low systematic error.

The higher contribution of repeated absorbance measurement of sample reaction to uncertainty of TIA measurement compared to repeated absorbance measurement of positive control reaction mixture was expected. Sample reaction mixture is more complex medium than the positive control reaction mixture having non- specific components which interact with coloration of L-BAPNA. According to [16] protein extraction using alkaline buffer is less specific resulting in extraction of non- specific components. However, repeated absorbance measurement of positive control reaction mixture had also considerable contribution to uncertainty of TIA measurement, indicating that part of uncertainty is derived from experimental conditions.

According to [17] divergence of absorbance measurements is in correlation with volume of reaction mixture and error of TIA measurement could be minimized if assaying is performed using $4 \mathrm{~mL}$ volume reaction mixtures or greater. Aliquot of the final sample solution used for preparation of the sample reaction mixture in microtiter plate method is only 45 $\mu \mathrm{l}$, however, [16] showed consistency of microtiter plate and AFNOR reference methods. According to [14] the smaller amounts of trypsin inhibitors can be measured by decreasing the volume of the reaction mixture while the concentration of reagents is kept unchanged. This was confirmed by [15] who compared TIA measurements of soybean variety Vojvodjanka obtained by microtiter plate method and modified AOCS method. AOCS method was performed with reaction mixture of $4 \mathrm{~mL}$ [14], [18] Although it was shown that reference and microtiter plate method give consistent TIA measurements, estimation of uncertainty factors that contribute to uncertainty of TIA measurement indicates that experimental conditions as well as the way of the preparation of the final sample solution could have a great impact on that uncertainty.

\section{CONCLUSION}

This study provides advancement in the processing of TIA results by revealing the sources that influence uncertainty of TIA measurement using a microtiter plate method. Estimated uncertainty of TIA measurement provided insight into the range of TIA values that should be expected. In addition, identified sources of measurement uncertainty also gave ability to compare results obtained by different methods. This could be of crucial importance considering the fact that there are three standards and many other similar methods for TIA measurement. Providing information 
on uncertainty sources that influence TIA measurements gives directions for improvement of methods used for TIA testing and contributes to improvement of TIA results management.

Acknowledgement: This research is a part of the Projects: "Developing vegetable varieties and hybrids for outdoor and indoor production" [TR-3103O]; "Physics and Chemistry with Ion Beams" [III45006], and "Joint research of measurements and effects of ionizing and $U V$ radiation in medicine and environmental protection" [III43011].

The authors are sincerely grateful to Ministry of Education and Science, Republic of Serbia, for supporting this study.

\section{REFERENCES}

1. J. Farkas, Irradiation of Dry Food Ingredients, Boca Raton (FL), USA: CRC Press, 1988

2. Food Irradiation: Principles and Applications, R.A. Molins, Ed., New York (NY), USA: John Wiley \& Sons, 2001

3. Food irradiation, S. Thorne, Ed., London, UK: Elsevier Applied Science, 1991

4. F. Roy, J.I. Boye and B.K. Simpson, "Bioactive Proteins and Peptides in Pulse Crops: Pea, Chickpea and Lentil," Food Res. Int., vol. 43, pp. 432-442, Mar. 2010

5. B. Bijina et al., "Protease Inhibitor from Moringa oleifera Leaves: Isolation, Purification, and Characterization”, Proc. Biochem., vol. 46, no. 12, pp. 2291-2300, Dec. 2011

6. J. Sabotič and J. Kos, "Microbial and Fungal Protease Inhibitors - Current and Potential Applications", Appl. Microbiol. Biot., vol 93, no. 4, pp. 1351-1375, Feb. 2012

7. E. Guillamón, M.M., Pedrosa, C. Burbano, C. Cuadrado, S.M. de Cortes and M. Muzquiz, "The Trypsin Inhibitors Present in Seed of Different Grain Legume Species and Cultivar," Food Chem., vol. 107, no. 1, pp. 68-74, Mar. 2008

8. A. Sattar, S.K. Durrani, F. Mahmood, A. Ahmad and I. Khan, "Effect of Soaking and Germination Temperatures on Selected Nutrients and Antinutrients of Mungbean", Food Chem., vol. 34, no. 2, pp. 111-120, 1989

9. A. Sattar, S. Atta, and M. A. Akhtar, "Effect of Radiation and Soaking on Trypsin Inhibitor and Protein Content of Chickpea (Cicer Arietinum L.)," Food / Nahrung, vol. 34 , no. 6, pp. 509-514, 1990
10. M.D.E.H. Farag, "Radiation Deactivation of Antinutritional Factors: Trypsin Inhibitor and Hemagglutinin in Soybeans," Egypt. J. Rad. Sci. Appl., vol. 6, pp. 207-215, 1989

11. Savezno ministarstvo za rad, zdravstvo i socijalnu politiku SRJ. (28 Avg. 1998). Pravilnik o uslovima pod kojima se mogu stavljati u promet namirnice i predmeti opšte upotrebe koji su konzervisani jonizujućim zračenjem ("Službeni list SRJ", br. 42/98). Federal Ministry for Labor, Health and Social Affairs, FR of Yugoslavia. (Aug. 28, 1998). Rulebook on Conditions under which Products Preserved with Ionizing Radiation Can be Released on the Market

12. European Commission. (Nov. 24, 2009). 52009XC1124(O2) List of Member States' authorisations of food and food ingredients which may be treated with ionising radiation (According to Article 4(6) of Directive 1999/2/EC of the European Parliament and of the Council on the approximation of the laws of the Member States concerning foods and food ingredients treated with ionising radiation).

13. Evaluation of measurement data - Guide to the expression of uncertainty in measurement, Working Group 1, Joint Committee for Guides in Metrology, 2008

14. K. Liu, P. Markakis, "An Improved Colorimetric Method for Determining Antitryptic Activity in Soybean Products," Cereal Chem., vol. 66, no. 5, pp. 415-422, Sep.-Oct. 1989

15. V. Župunski, Z. Mitrović, Z. Nikolić, G. Petrović, G. Tamindžić, Đ. Karagić, "Uncertainty Analysis of the Microtiter Plate Method for Determining Trypsin Inhibitor Activity," Accred. Q. A., vol. 21, no. 2, pp. 151160, Apr. 2016

16. D. Page, L. Quillien and G. Duc, "Trypsin Inhibitory Activity Measurement: Simplifications of the Standard Procedure Used for Pea Seed," Crop Sci., vol. 40, no. 5, pp. 1482-1485, 2000

17. G. E. Hamerstrand, L. T. Black and J. D. Glover, "Trypsin Inhibitors in Soy Products: Modification of the Standard Analytical Procedure". Cereal Chem., vol. 58, no. 1, pp. 42-45, Jan.-Feb. 1981

18. M.B. Pesic, B.V. Vucelic-Radovic, M.B. Barac, S.P. Stanojevic and V.A. Nedovic, "Influence of Different Genotypes on Trypsin Inhibitor Levels and Activity in Soybeans," Sensors, vol. 7, no. 1, pp. 67-74, Jan. 2007 